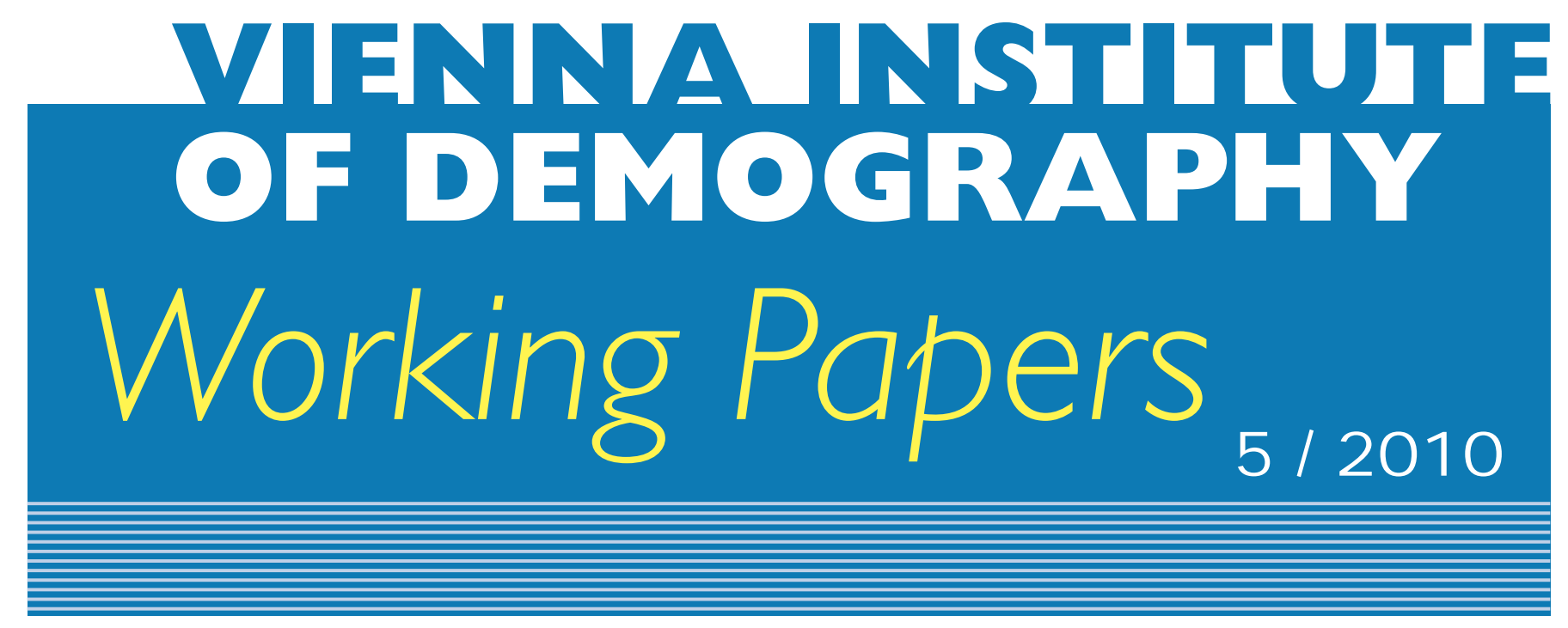

Theresa Grafeneder-W eissteiner and Klaus Prettner

\title{
Agglomeration Processes in Ageing Societies
}




\begin{abstract}
This article investigates agglomeration processes in ageing societies by introducing an overlapping generation structure into a New Economic Geography model. Whether higher economic integration leads to spatial concentration of economic activity crucially hinges on the economies' demographic properties. While population aging as represented by declining birth rates strengthens agglomeration processes, declining mortality rates weaken them. This is due to the fact that we allow for nonconstant population size. In particular, we show that population growth acts as an important dispersion force that augments the distributional effects on agglomeration processes resulting from the turnover of generations.
\end{abstract}

JEL classification: R12, J10, F15, C61

\title{
Keywords
}

Agglomeration, population aging, population.

\section{Authors}

Theresa Grafeneder-Weissteiner: Vienna University of Economics and Business Administration, Department of Economics, Augasse 2-6, A-1090, Vienna, Austria; email: tgrafene@wu-wien.ac.at; tel: +431313364088

Klaus Prettner: Vienna Institute of Demography, Austrian Academy of Sciences, Wohllebengasse 12-14, A-1040, Vienna, Austria; email: klaus.prettner@oeaw.ac.at; tel: +43 1 515817729

\section{Acknowledgements}

We thank Harald Fadinger (University of Vienna), Ingrid Kubin (WU), Alexia Prskawetz (Vienna University of Technology), Michael Rauscher (University of Rostock), Gerhard Sorger (University of Vienna), Stefan Wrzaczek (Vienna University of Technology), and the conference participants at the WWTF (Vienna Science and Technology Fund) Workshop "Agglomeration Processes in Ageing Societies" 2010 for helpful comments and suggestions. The paper was prepared within the research project "Agglomeration Processes in Ageing Societies" funded by the WWTF in its "Mathematics and..." Call 2007. 


\title{
Agglomeration processes in ageing societies
}

\author{
Theresa Grafeneder-Weissteiner and Klaus Prettner
}

\section{Introduction}

With the ongoing worldwide economic integration during the last decades, economists' and policy makers' interest in the location of economic activity has dramatically risen (see e.g. Fujita and Thisse (2002) and the World Bank (2009)'s development report on economic geography). Within the European Union for example, regional cohesion policies targeted at a more equal spatial allocation of resources are on top of the political agenda. At the same time, as illustrated in the special report on ageing populations of The Economist (2009), demographic change has been creating serious economic challenges for industrialized countries. In particular, declining fertility has caused upward shifts in the mean age of most countries' populations while simultaneously reducing population growth rates (see Eurostat (2009) and United Nations (2007)).

Up to now, these two issues have been analysed independently of each other, with the New Economic Geography (NEG) addressing the impact of deeper economic integration on the spatial concentration of productive factors and overlapping generation (OLG) models investigating the effects of demography on macroeconomic aggregates. This division is unfortunate since both demographic change and economic integration crucially influence demand patterns which themselves are - via the returns to productive factors - decisive for the spatial distribution of economic activity. Explaining agglomeration processes without accounting for demographic developments thus misses a fundamental point.

Grafeneder-Weissteiner and Prettner (2009) have made a first step toward closing this gap by merging these two research strands and for the first time providing a unified framework within which the linkage between demographic change and agglomeration can be accurately analysed. In particular, the have shown that introducing an overlapping generation setting where individuals face lifetime uncertainty considerably reduces agglomeration tendencies. By equalizing the birth to the death rate, their framework, however, can only analyse the effects of changes in the population age structure while ignoring the impacts of varying population growth due to demographic change. Since declining fertility leads to decreasing population growth rates while lower mortality rates imply higher population growth, it is essential to reassess the impact of demographic change on the location of industries in a setting with nonconstant population size. Having this purpose in mind, the model presented in this paper extends Grafeneder-Weissteiner and Prettner (2009)'s approach by allowing for nonequal birth and mortality rates and thus growing populations. 
In particular, we generalize Baldwin (1999)'s constructed capital model by incorporating Buiter (1988)'s overlapping generation structure to arrive at a NEG framework featuring both population ageing and varying population size.

In Baldwin (1999)'s constructed capital model concentration of economic activity is explained via a demand-linked circular causality whose pro-agglomerative effect is strongest for high levels of economic integration. In particular, with interregionally immobile capital, a higher capital stock raises capital income and thus expenditures which leads to an increase in the capital rental rate and therefore to further capital accumulation. Since higher capital accumulation is typically associated with medium-run growth, this tworegion neoclassical growth framework illustrates how economic integration can lead to the emergence of "rust" and "boom belts". Its intertemporally optimized saving features moreover allow an easy incorporation of overlapping generations with individuals that face a positive probability of death and differ with respect to their age. By using Buiter (1988)'s overlapping generation structure, demographic change capturing both changes in the population age structure and/or in the population growth rate can be analysed via variations in either the birth or the mortality rates. In particular, we can analyse a situation of population ageing and declining population growth rates as recently faced by many industrialized countries. Overall, our modelling strategy does not only allow us to accurately analyse agglomeration processes in ageing societies but also provides us with a more convincing description of reality compared to Baldwin (1999)'s setting with one infinitely lived individual.

Our results show that the possibility of agglomeration crucially hinges on the economies' demographic properties, i.e. on the birth and mortality rate. While declining birth rates strengthen agglomeration processes, declining mortality rates weaken them. These differential effects on the stability properties of the symmetric equilibrium are due to the opposite impact of changes in the birth and mortality rate on the population size. Lower birth rates decrease the population growth rate, while lower mortality rates increase it. Since population growth weakens the wealth and thus expenditure increase due to a higher capital stock, it acts as an important dispersion force. For declining birth rates, the population growth based channel therefore strengthens the pro-agglomerative effects of a lower turnover of generations first identified by Grafeneder-Weissteiner and Prettner (2009), while for declining mortality rates the implied increase in the population growth rate counteracts and even dominates the impacts via the turnover channel.

The remainder of the paper is structured as follows. Section 2 presents the model framework and derives the equilibrium laws of motion for capital and expenditures. Section 3 first verifies the existence of a symmetric long-run equilibrium and then investigates its stability properties. In doing so, we can not only analyse agglomeration processes in ageing societies but also isolate the population growth based effect on agglomeration. Finally, section 4 contains concluding remarks and sketches possible lines of further research. 


\section{The model}

This section describes how we integrate Buiter (1988)'s overlapping generation structure with growing populations into the constructed capital framework of Baldwin (1999) to arrive at a NEG framework allowing for both population ageing and varying population size.

There are two symmetric regions or countries, denoted as $H$ (home) and $F$ (foreign $)^{1}$, with identical production technologies, trade costs, preferences of individuals, labour endowments and demographic structures. Each region has three economic sectors (agriculture, manufacturing and investment) with two immobile factors (labour $L$ and capital $K$ ) at their disposal.

The homogeneous agricultural good, which is also the numeraire good and denoted as $z$, is produced under constant returns to scale in a perfectly competitive market using labour as the only input with, by choice of units, an input coefficient of one. It can be freely traded between the two regions.

Manufacturing firms behave as in the monopolistic competition framework of Dixit and Stiglitz (1977) and therefore produce horizontally differentiated varieties, $m$, with one unit of capital as fixed input and a variable per unit requirement of $a_{m}$ units of labour. A continuum of varieties $i \in\left(0, V_{H}\right]$ is produced at home, whereas a continuum of varieties $j \in\left(0, V_{F}\right]$ is manufactured in the foreign region. In contrast to the agricultural good, trade of manufactures involves iceberg transport costs such that $\varphi \geq 1$ units of a certain good have to be shipped in order to sell one unit abroad (see e.g. Baldwin et al. (2003)). Firms thus face an increasing returns to scale production technology with an associated cost function $\pi+w a_{m} Y_{m}(i)$, where $\pi$ is the capital rental rate representing the fixed cost, $w$ is the wage per efficiency unit of labour and $Y_{m}(i)$ is total output of one manufacturing good producer.

In the Walrasian investment sector, capital, i.e. machines, are produced using labour as the only input with an input coefficient of $a_{i}$. Wages of the workers are paid out of individuals' savings. Following Baldwin (1999), a share $\delta>0$ of the capital stock depreciates at each instant.

Concerning the overlapping generation structure of our model economy, we closely follow Buiter (1988). We assume that at each point in time, $\tau \in[0, \infty)$, a large cohort consisting of new individuals is born. Newborns receive no bequests and thus start their lives without any wealth. Each individual's time of death is stochastic with an exponential probability density function parameterized by the constant instantaneous mortality rate $\mu>0$. Normalizing initial population size $N(0)$ to one, the size of the cohort born at $t_{0}$ at a certain point in time $\tau$ is $N\left(t_{0}, \tau\right)=\beta e^{\beta t_{0}} e^{-\mu \tau}$ (see appendix A.1) ${ }^{2}$, where $\beta>0$ is

\footnotetext{
${ }^{1}$ If further distinction is needed, foreign variables are moreover indicated by an asterisk. In particular, the superscript $F$ denotes that a good was produced in the foreign region, whereas the asterisk indicates that it is consumed in the foreign region. In what follows, emphasis will be on the home region. The corresponding expressions for the foreign region can be derived by symmetry.

${ }^{2}$ In what follows the first time index of a variable will refer to the birth date, whereas the second will
} 
the constant birth rate. Consequently, total population size at time $\tau$ is given by

$$
\begin{aligned}
N(\tau) & =\int_{-\infty}^{\tau} N\left(t_{0}, \tau\right) d t_{0} \\
& =\int_{-\infty}^{\tau} \beta e^{\beta t_{0}} e^{-\mu \tau} d t_{0} \\
& =e^{(\beta-\mu) \tau},
\end{aligned}
$$

where we denote its growth rate as $n \equiv \beta-\mu$.

Since there is no heterogeneity between members of the same cohort, each cohort can be described by one representative individual, who inelastically supplies her efficiency units of labour $l$ on the labour market with perfect mobility across sectors but immobility between regions. Finally, as in Yaari (1965), individuals can insure themselves against the risk of dying with positive assets by buying actuarial notes of a fair life insurance company which are cancelled upon their death.

\subsection{Individual consumption behaviour}

Preferences over the agricultural good and a CES composite of the manufacturing varieties are Cobb-Douglas. The representative individual of the cohort born at $t_{0}$ chooses at each instant $\tau>t_{0}$ consumption of the agricultural good, $c_{z}\left(t_{0}, \tau\right)$, consumption of varieties produced at home, $c_{m}^{H}\left(i, t_{0}, \tau\right)$, and consumption of varieties produced abroad, $c_{m}^{F}\left(j, t_{0}, \tau\right)$, to maximize her expected lifetime utility at time $t_{0}{ }^{3}$

$$
U\left(t_{0}, t_{0}\right)=\int_{t_{0}}^{\infty} e^{-(\rho+\mu)\left(\tau-t_{0}\right)} \ln \left[\left(c_{z}\left(t_{0}, \tau\right)\right)^{1-\xi}\left(c_{m}^{a g g}\left(t_{0}, \tau\right)\right)^{\xi}\right] d \tau
$$

where $\rho>0$ is the rate of pure time preference, $0<\xi<1$ is the manufacturing share of consumption and

$$
c_{m}^{a g g}\left(t_{0}, \tau\right) \equiv\left[\int_{0}^{V_{H}(\tau)}\left(c_{m}^{H}\left(i, t_{0}, \tau\right)\right)^{\frac{\sigma-1}{\sigma}} d i+\int_{0}^{V_{F}(\tau)}\left(c_{m}^{F}\left(j, t_{0}, \tau\right)\right)^{\frac{\sigma-1}{\sigma}} d j\right]^{\frac{\sigma}{\sigma-1}}
$$

represents consumption of the CES composite with $\sigma>1$ being the elasticity of substitution between varieties.

The wealth constraint of a representative individual is given by

$$
\dot{k}\left(t_{0}, \tau\right)=\frac{w(\tau) l+\pi(\tau) k\left(t_{0}, \tau\right)-e\left(t_{0}, \tau\right)}{w(\tau) a_{i}}+\mu k\left(t_{0}, \tau\right)-\delta k\left(t_{0}, \tau\right)
$$

where $k\left(t_{0}, \tau\right)$ is the individual capital stock and $e\left(t_{0}, \tau\right)$ are individual expenditures for

indicate a certain point in time.

${ }^{3}$ Equation (2) can be easily derived by calculating expected lifetime utility, where the date of death is a random variable with an exponential probability density function parameterized by a constant instantaneous mortality rate $\mu$. 
consumption defined as

$$
\begin{aligned}
e\left(t_{0}, \tau\right) \equiv & p_{z}(\tau) c_{z}\left(t_{0}, \tau\right)+\int_{0}^{V_{H}(\tau)} p_{m}^{H}(i, \tau) c_{m}^{H}\left(i, t_{0}, \tau\right) d i+ \\
& \int_{0}^{V_{F}(\tau)} p_{m, \varphi}^{F}(j, \tau) c_{m}^{F}\left(j, t_{0}, \tau\right) d j .
\end{aligned}
$$

Here $p_{z}(\tau)$ is the price of the agricultural $\operatorname{good}, p_{m}^{H}(i, \tau)$ the price of a manufactured variety produced at home and $p_{m, \varphi}^{F}(j, \tau)$ the price of a manufactured variety produced abroad with the subscript $\varphi$ indicating the dependence on transport costs. This wealth constraint displays the structure of the investment sector by showing that individual savings, defined as income minus consumption expenditures, are transformed into capital.

The law of motion for capital given in equation (3) is based on Yaari (1965)'s full insurance result implying that individuals save solely in the form of actuarial notes from the life insurance company, whose fair rate exceeds the market rate of return on capital, $\frac{\pi(\tau)}{w(\tau) a_{i}}-\delta$, by $\mu$ (see Yaari (1965)).

The individual's utility optimization problem can be solved by applying a three stage procedure. ${ }^{4}$ The first stage analyses the dynamic savings-expenditure decision and results in the Euler equation for the representative individual of the cohort born at $t_{0}$

$$
\frac{\dot{e}\left(t_{0}, \tau\right)}{e\left(t_{0}, \tau\right)}=\frac{\pi(\tau)}{a_{i} w(\tau)}-\delta-\rho
$$

Stage two and three finally deal with the static optimal consumption allocation between the CES composite and the agricultural good as well as with the allocation of consumption to each of the varieties. Altogether this leads to the following demand functions for the agricultural good and for each of the manufactured varieties

$$
\begin{aligned}
c_{z}\left(t_{0}, \tau\right) & =\frac{(1-\xi) e\left(t_{0}, \tau\right)}{p_{z}(\tau)}, \\
c_{m}^{H}\left(i, t_{0}, \tau\right) & =\frac{\xi e\left(t_{0}, \tau\right)\left(p_{m}^{H}(i, \tau)\right)^{-\sigma}}{\left[\int_{0}^{V_{H}(\tau)}\left(p_{m}^{H}(i, \tau)\right)^{1-\sigma} d i+\int_{0}^{V_{F}(\tau)}\left(p_{m, \varphi}^{F}(j, \tau)\right)^{1-\sigma} d j\right]}, \\
c_{m}^{F}\left(j, t_{0}, \tau\right) & =\frac{\xi e\left(t_{0}, \tau\right)\left(p_{m, \varphi}^{F}(j, \tau)\right)^{-\sigma}}{\left[\int_{0}^{V_{H}(\tau)}\left(p_{m}^{H}(i, \tau)\right)^{1-\sigma} d i+\int_{0}^{V_{F}(\tau)}\left(p_{m, \varphi}^{F}(j, \tau)\right)^{1-\sigma} d j\right]} .
\end{aligned}
$$

\subsection{Aggregation}

Due to the overlapping generation structure, our model setup does not feature one single representative individual. Corresponding to any individual variable we thus define population aggregates that follow from aggregating up over all cohorts. For capital and

\footnotetext{
${ }^{4}$ For details of the derivations see Grafeneder-Weissteiner and Prettner (2009)
} 
expenditures, these aggregation rules are formally given by

$$
\begin{aligned}
K(t) & \equiv \int_{-\infty}^{t} k\left(t_{0}, t\right) N\left(t_{0}, t\right) d t_{0}, \\
& =\beta e^{-\mu t} \int_{-\infty}^{t} k\left(t_{0}, t\right) e^{\beta t_{0}} d t_{0}, \\
E(t) & \equiv \int_{-\infty}^{t} e\left(t_{0}, t\right) N\left(t_{0}, t\right) d t_{0}, \\
& =\beta e^{-\mu t} \int_{-\infty}^{t} e\left(t_{0}, t\right) e^{\beta t_{0}} d t_{0},
\end{aligned}
$$

where $K(t)$ is the aggregate capital stock and $E(t)$ denotes aggregate consumption expenditures. ${ }^{5}$

For each population aggregate variable $X(t)$, the corresponding quantity per capita is defined by $\tilde{x}(t)=X(t) e^{-n t}$ (see Buiter (1988)). Using this notational convention, we derive in appendix A.2 the following laws of motion for per capita expenditures $\tilde{e}(t)$ and per capita capital $\tilde{k}(t)$

$$
\begin{aligned}
& \dot{\tilde{e}}(t)=\left[\frac{\pi(t)}{w(t) a_{i}}-\delta-\rho\right] \tilde{e(t)}-\beta(\rho+\mu) a_{i} w(t) \tilde{k}(t), \\
& \dot{\tilde{k}}(t)=\left(\frac{\pi(t)}{w(t) a_{i}}-\delta-\beta+\mu\right) \tilde{k}(t)+\frac{\tilde{l}}{a_{i}}-\frac{\tilde{e}(t)}{w(t) a_{i}},
\end{aligned}
$$

where analogous equations hold in the foreign region. In contrast to the setting with a constant population size of Grafeneder-Weissteiner and Prettner (2009), where the mortality rate only enters the aggregate Euler equation, demographic parameters appear in both laws of motion. In particular, the law of motion of per capita expenditures differs from the individual Euler equation by $-\beta(\rho+\mu) a_{i} w(t) \tilde{k}(t)$. Rewriting equation (10) as (see also appendix A.2)

$$
\frac{\dot{\tilde{e}}(t)}{\tilde{e}(t)}=\frac{\dot{e}\left(t_{0}, \tau\right)}{e\left(t_{0}, \tau\right)}-\beta \frac{\tilde{e}(t)-e(t, t)}{\tilde{e}(t)}
$$

sheds more light on how to explain the emergence of this additional term. As explained in detail in Grafeneder-Weissteiner and Prettner (2009) for the case of a constant population size, the difference between individual and per capita consumption expenditure growth is due to the distributional effects of the turnover of generations. At each instant in time a fraction $\mu$ of wealthier individuals with high consumption expenditures dies ${ }^{6}$ and is replaced by a fraction $\beta$ of newborns without capital holdings and thus lower consumption expenditures. This continually ongoing process captured by the difference between average consumption expenditures $\tilde{e}(t)$ and consumption expenditures of newborns $e(t, t)$ slows down per capita consumption expenditure growth (aggregate economy average) relative to individual consumption expenditure growth.

\footnotetext{
${ }^{5}$ Note that aggregate efficiency units of labour are accordingly given by $L(t)=l e^{(\beta-\mu) t}$.

${ }^{6}$ Due to the law of large numbers, the individual probability of dying is equal to the fraction of individuals who die at each instant.
} 
Whereas both the birth and the mortality rate strengthen the turnover correction term and thus decrease per capita expenditure growth ${ }^{7}$, their effects on the per capita law of motion of capital are of opposite sign. Obviously, it is in fact the population growth rate $n=\beta-\mu$ that enters the per capita law of motion for capital. A higher $n$ decreases per capita accumulation by decreasing the market rate of return on capital. It is not surprising that this last relationship will become crucial when investigating the effects of population growth on agglomeration tendencies. To do so, we first have to determine the equilibrium factor prices resulting out of profit maximization in order to arrive at a dynamic system for expenditures and capital that fully describes the evolution of our economy.

\subsection{Profit maximization}

Profit maximization and perfect competition in the agricultural sector imply that the price of an agricultural good equals its marginal cost. Since labour is perfectly mobile across sectors, the wage rate in the economy is thus pinned down by the price of the agricultural good. Free trade equalizes this price and thus wages across regions as long as each region produces some agricultural output which will be assumed from now on. ${ }^{8}$ We thus have

$$
w(t)=w^{*}(t)=1
$$

since the agricultural good has been chosen as the numeraire.

Profit maximization in the monopolistically competitive manufacturing sector ${ }^{9}$ yields

$$
\begin{aligned}
p_{m}^{H}(i, t) & =\frac{\sigma}{\sigma-1} w(t) a_{m}, \\
p_{m, \varphi}^{F}(i, t) & =p_{m}^{H}(i, t) \varphi .
\end{aligned}
$$

Prices are equal to a constant markup over marginal costs and mill pricing is optimal, i.e. the only difference between prices in the two regions is due to transport costs (see e.g. Baldwin et al. (2003)).

Since there is free entry into the manufacturing sector, pure profits will be driven down to zero. Consequently, the capital rental rate, which represents the fixed costs of each manufacturing firm, is pinned down by the level of operating profits. Using optimal prices given in equations (14) and (15) together with equations (6) and (7) and redefining global quantities and regional share variables ${ }^{10}$ gives operating profits and thus capital

\footnotetext{
${ }^{7}$ Recall that by equalizing the birth to the death rate, Grafeneder-Weissteiner and Prettner (2009) were not able to differentiate between the impact of varying birth and mortality rates, i.e. only $\mu$ appeared in the turnover correction term.

${ }^{8}$ See Baldwin (1999) for details on this assumption.

${ }^{9}$ See again Grafeneder-Weissteiner and Prettner (2009) for details of the derivations.

${ }^{10}$ Note that the number of varieties in the home region $V_{H}(t)$ is equal to the capital stock at home $K(t)$ because one variety exactly requires one unit of capital as fixed input (analogously $K^{*}(t) \equiv V_{F}(t)$ ).
} 
rental rates as ${ }^{11}$

$$
\begin{aligned}
\pi & =\left(\frac{\theta_{E}}{\theta_{K}+\phi\left(1-\theta_{K}\right)}+\frac{\left(1-\theta_{E}\right) \phi}{\phi \theta_{K}+1-\theta_{K}}\right)\left(\frac{\xi E^{W}}{\sigma K^{W}}\right), \\
\pi^{*} & =\left(\frac{1-\theta_{E}}{1-\theta_{K}+\phi \theta_{K}}+\frac{\theta_{E} \phi}{\phi\left(1-\theta_{K}\right)+\theta_{K}}\right)\left(\frac{\xi E^{W}}{\sigma K^{W}}\right),
\end{aligned}
$$

where $\phi \equiv \varphi^{1-\sigma}$ measures trade openness between the two regions with $\phi=0$ indicating prohibitive trade barriers and $\phi=1$ free trade. World expenditures are defined as $E^{W} \equiv$ $E+E^{*}$ and the world capital stock as $K^{W} \equiv K+K^{*}$ with $\theta_{K}$ and $\theta_{E}$ referring to the home shares of these quantities.

At the symmetric equilibrium with $\theta_{K}=1 / 2$ and $\theta_{E}=1 / 2$, shifting expenditure to home $\left(d \theta_{E}>0\right)$ raises $\pi$ and lowers $\pi^{*}$ since it increases the home market size. A higher expenditure share therefore promotes agglomeration of capital at home because capital accumulates where the rental rate is higher and decumulates in the other region. Production shifting to home $\left(d \theta_{K}>0\right)$, on the other hand, has the opposite effects because it increases competition in the home market (local competition effect). The relative strength of both forces determines whether agglomeration processes set in in a framework ignoring demographic change. In particular, Baldwin (1999) shows that the pro-agglomerative force dominates for sufficiently high levels of trade openness.

Grafeneder-Weissteiner and Prettner (2009) have already shown that an overlapping generation structure with lifetime uncertainty introduces a crucial third force working via the turnover correction term. Since a higher capital stock in one region implies that dying individuals are on average richer, the distributional effects are more severe relative to the region with the lower capital stock. Consequently, the turnover of generations acts as an anti-agglomerative force by reducing relative aggregate consumption expenditure growth of the region with the higher capital stock. The crucial question to be analysed in the following sections is how the introduction of a nonconstant population size, i.e. $\mu \neq \beta$, affects this linkage between demographic change and agglomeration. In particular, we investigate how population ageing as represented by declining fertility rates impacts upon the spatial distribution of economic activity by simultaneously taking into account the associated changes in the population growth rate.

\section{The impact of demographic change on agglomeration}

To assess whether and how demographic change impacts upon agglomeration processes we analyse the stability properties of the symmetric equilibrium more thoroughly. If it turns out that this steady state is unstable, then any slight perturbation will lead us away from an equal distribution of capital and expenditures and thus result in agglomeration processes.

\footnotetext{
${ }^{11}$ We ignore time arguments here. Note, moreover, that $\xi=\xi^{*}$ and $\sigma=\sigma^{*}$ due to symmetry between regions. For further details of the derivations see again Grafeneder-Weissteiner and Prettner (2009).
} 


\subsection{Long-run equilibrium}

Using the rental rates from equations (16) and (17) reformulated as functions of the variables $\tilde{e}, \tilde{e}^{*}, \tilde{k}$ and $\tilde{k}^{*}$ (see appendix B) as well as the equilibrium wages from equation (13) in the per capita laws of motion of capital and consumption expenditures (10) and (11) yields the following four dimensional system ${ }^{12}$

$$
\begin{aligned}
\dot{\tilde{e}} & =\left[\left(\frac{\tilde{e}}{\tilde{\tilde{k}}+\phi \tilde{k}^{*}}+\frac{\tilde{e}^{*} \phi}{\phi \tilde{k}+\tilde{k}^{*}}\right)\left(\frac{\xi}{a_{i} \sigma}\right)-\delta-\rho\right] \tilde{e}-\beta(\rho+\mu) a_{i} \tilde{k}, \\
\dot{\tilde{e}}^{*} & =\left[\left(\frac{\tilde{e}^{*}}{\tilde{k}^{*}+\phi \tilde{k}}+\frac{\tilde{e} \phi}{\phi \tilde{k}^{*}+\tilde{k}}\right)\left(\frac{\xi}{a_{i} \sigma}\right)-\delta-\rho\right] \tilde{e}^{*}-\beta(\rho+\mu) a_{i} \tilde{k}^{*}, \\
\dot{\tilde{k}} & =\left[\left(\frac{\tilde{e}}{\tilde{\tilde{k}}+\phi \tilde{k}^{*}}+\frac{\tilde{e}^{*} \phi}{\phi \tilde{k}+\tilde{k}^{*}}\right)\left(\frac{\xi}{a_{i} \sigma}\right)-\delta-\beta+\mu\right] \tilde{k}+\frac{\tilde{l}}{a_{i}}-\frac{\tilde{e}}{a_{i}}, \\
\dot{\tilde{k}}^{*} & =\left[\left(\frac{\tilde{e}}{\tilde{e}^{*}+\phi \tilde{k}}+\frac{\tilde{e} \phi}{\phi \tilde{k}^{*}+\tilde{k}}\right)\left(\frac{\xi}{a_{i} \sigma}\right)-\delta-\beta+\mu\right] \tilde{k}^{*}+\frac{\tilde{l}}{a_{i}}-\frac{\tilde{e}^{*}}{a_{i}} .
\end{aligned}
$$

These four differential equations in the variables $\tilde{e}, \tilde{e}^{*}, \tilde{k}$ and $\tilde{k}^{*}$ fully describe the dynamics of our NEG model with overlapping generations and a nonconstant population size. Note that they nest both the Baldwin (1999) set-up with $\beta=\mu=0$ as well as the GrafenederWeissteiner and Prettner (2009) framework with $\beta=\mu>0$ as special cases. The latter will turn out to be particularly useful when we try to isolate the effect of population growth on agglomeration tendencies.

Inserting the symmetric outcome $\tilde{e}=\tilde{e}^{*}$ and $\tilde{k}=\tilde{k}^{*}$ into the above system indeed reveals that it is a steady state with the equilibrium values given by ${ }^{13}$

$$
\begin{aligned}
& \overline{\tilde{e}}=\frac{\tilde{l} \sigma((\delta-\mu) \sqrt{\sigma}(A+B)+\beta((\delta+\rho-2 \mu) \sigma+2(\mu+\rho) \xi+A \sqrt{\sigma}))}{2((\delta-\mu) \sigma+\beta \xi)((\beta+\delta+\rho) \sigma-(\mu+\rho) \xi)}, \\
& \overline{\tilde{k}}=\frac{\tilde{l}((\rho \sigma-\sqrt{\sigma} A)(\sigma-\xi)+2 \sigma \xi(\beta-\mu)+\delta \sigma(\sigma+\xi))}{2 a_{i}((\delta-\mu) \sigma+\beta \xi)((\beta+\delta+\rho) \sigma-(\mu+\rho) \xi)},
\end{aligned}
$$

where $A \equiv \sqrt{\sigma(\delta+\rho)^{2}+4 \beta(\mu+\rho) \xi}$ and $B \equiv(\delta+\rho) \sqrt{\sigma}$.

\subsection{Formal stability analysis}

To analyse the stability properties of the symmetric equilibrium we first linearise the nonlinear dynamic system given in equations (18), (19), (20) and (21) around the symmetric equilibrium (22) and (23), and then evaluate the eigenvalues of the corresponding $4 \times 4$

\footnotetext{
${ }^{12}$ We again suppress time arguments here. Note, moreover, that we have $\tilde{l}=\tilde{l}^{*}, \mu=\mu^{*}, \beta=\beta^{*} a_{i}=a_{i}^{*}$, $\delta=\delta^{*}$ and $\rho=\rho^{*}$ due to symmetry between regions.

${ }^{13}$ These and most of the following results were derived with Mathematica. The corresponding files are available from the authors upon request. Note also that we restrict attention to the economically meaningful solution pair, i.e. where consumption and capital is positive for plausible parameter values.
} 
Jacobian matrix

$$
J_{\text {sym }}=\left(\begin{array}{ll}
J_{1} & J_{2} \\
J_{3} & J_{4}
\end{array}\right),
$$

where the four symmetric $2 \times 2$ sub-matrices $J_{i}$ for $i=1, \ldots, 4$ are given in appendix $\mathrm{C}$ (for this classical approach of stability analysis see e.g. the appendix on mathematical methods in Barro and Sala-i-Martin (2004)). Solving the characteristic equation yields the following four eigenvalues

$$
\begin{aligned}
e i g 1 & =\frac{1}{2}\left(r_{1}-\sqrt{\operatorname{rad}_{1}}\right), \\
e i g 2 & =\frac{1}{2}\left(r_{1}+\sqrt{\operatorname{rad}_{1}}\right), \\
e i g 3 & =\frac{1}{2(\phi+1)^{2} \sqrt{\sigma}}\left(r_{2}-\sqrt{r a d_{2}}\right), \\
e i g 4 & =\frac{1}{2(\phi+1)^{2} \sqrt{\sigma}}\left(r_{2}+\sqrt{\operatorname{rad}_{2}}\right),
\end{aligned}
$$

where

$$
\begin{aligned}
r_{1} \equiv & \frac{A}{\sqrt{\sigma}}-\beta-\delta+\mu \\
\operatorname{rad}_{1} \equiv & \left(\frac{A}{\sqrt{\sigma}}+\beta+\delta-\mu\right)^{2}+\frac{(\sigma-\xi)\left((A+B)^{2}+4 \beta(\mu+\rho) \xi\right)}{\sigma \xi}, \\
r_{2} \equiv & \sqrt{\sigma}\left(\mu-\beta(\phi+1)^{2}-\delta\left(2 \phi^{2}+\phi+1\right)+\phi(\mu(\phi+2)-\rho(\phi-1))\right) \\
& +A(3 \phi+1), \\
\operatorname{rad}_{2} \equiv & \left(A(\phi-1)+\left(\mu(\phi+1)^{2}-\beta(\phi+1)^{2}+\delta(\phi-1)+\phi(\phi+3) \rho\right) \sqrt{\sigma}\right)^{2}+ \\
& \frac{(\phi+1)((\phi+1) \sigma+(\phi-1) \xi)\left(4 \beta(\mu+\rho) \xi(\phi+1)^{2}+(\phi-1)^{2}(A+B)^{2}\right)}{\xi} .
\end{aligned}
$$

The nature and signs of these eigenvalues fully characterize the system's local dynamics around the symmetric equilibrium. First it is easily established that all four eigenvalues are real since both $\mathrm{rad}_{1}$ and $\mathrm{rad}_{2}$ are nonnegative for all possible parameter values. ${ }^{14}$ Turning to the signs of the eigenvalues, the analysis is more involved. Indeed, the above eigenvalues can be used to assess the effects of demographic change on the stability properties of the symmetric equilibrium for all three cases of growing population, i.e. $\beta>\mu$, shrinking population, i.e. $\beta<\mu$, and a constant population size, i.e. $\beta=\mu$. The last scenario has already been investigated in Grafeneder-Weissteiner and Prettner (2009), who show that in this case eigenvalue three is decisive for the stability properties of the symmetric equilibrium. In particular, the system is saddle path stable only for parameter ranges that yield a negative eigenvalue three.

For the case of positive population growth, i.e. $\beta>\mu$, eigenvalue three retains its crucial role, which becomes clear when checking the signs of the remaining other eigen-

\footnotetext{
${ }^{14}$ Recall the parameter ranges $\mu>0, \beta>0, \delta>0, \sigma>1, \rho>0,0<\xi<1$ and $0 \leq \phi \leq 1$ which also imply that $A>0$ and $B>0$. In particular, note that $\sigma>\xi$.
} 
values. Note first, that the sign of $r_{1}$ is ambiguous. ${ }^{15}$ As far as eigenvalue 1 is concerned, it is for sure nonpositive as long as $r_{1}<0$. For the case of $r_{1}>0$, we can show that $r_{1}^{2}<\operatorname{rad}_{1}$ implying that eigenvalue 1 never gets positive. The last inequality moreover yields that eigenvalue 2 , on the other hand, is always nonnegative. ${ }^{16}$ Finally, turning to the sign of eigenvalue 4 , note first that $r_{2}$ again does not have an unambiguous sign. ${ }^{17}$ For $r_{2} \geq 0$, eigenvalue 4 is for sure nonnegative. Using Mathematica, it is, however, possible to show that $r_{2}+\sqrt{\mathrm{rad}_{2}}>0$ even for negative $r_{2}$ implying that eigenvalue 4 is nonnegative for all possible parameter ranges. Summarizing, we have two positive and one negative eigenvalue. Both for the case of constant population size and population growth the symmetric equilibrium thus becomes unstable for parameter values, and in particular birth and mortality rates, that yield a positive eigenvalue 3 .

For the case of population shrinking, i.e. $\mu>\beta$, only eigenvalue 2 and 4 have unambiguous signs. Note first, that $r_{1}>0$ if $\mu>\beta$ which immediately proves the nonnegativity of eigenvalue 2. Similarly, it can be shown that $r_{2}>0$ if $\mu>\beta^{18}$ implying that eigenvalue 4 is always nonnegative as well. Thus, for the case of population shrinking, agglomeration processes will set in for birth or mortality rates for which at least either eigenvalue 1 or eigenvalue 3 is positive.

Since the above findings illustrate that demographic change, i.e. variations in $\beta$ and $\mu$, can only influence the stability properties of the symmetric equilibrium via eigenvalue 3 for the case of $\beta \geq \mu$ and eigenvalues 1 and 3 for $\beta<\mu$, it is immediate to investigate them more thoroughly. Figures 1 and 2, which plot the contour lines of eigenvalues 1 and 3 for different birth and mortality rates ${ }^{19}$, show that both of them switch their sign depending on the economies' demographic parameters. For the case of population shrinking, i.e. $\beta<\mu$, note moreover that for all combinations of $\beta$ and $\mu$ for which eigenvalue 3 switches sign, eigenvalue 1 is still negative. These observations immediately result in the following proposition.

Proposition 1. The possibility of agglomeration crucially hinges on the economies' demographic properties. In particular, both the birth and the mortality rate are decisive for the stability properties of the symmetric equilibrium.

Proof. See figures 1 and 2 and above arguing on the eigenvalues' signs.

\footnotetext{
${ }^{15}$ In particular, $r_{1}$ becomes negative for sufficiently high $\beta$.

${ }^{16}$ For $r_{1}>0$ this follows trivially, $r_{1}^{2}<\operatorname{rad}_{1}$ also shows it for $r_{1}<0$.

${ }^{17}$ In particular, $r_{2}$ becomes negative for sufficiently high $\beta$.

${ }^{18}$ This follows from rewriting

$$
r_{2}=\underbrace{A(3 \phi+1)+\sqrt{\sigma}\left(-\delta\left(2 \phi^{2}+\phi+1\right)+\phi \rho(1-\phi)\right)}_{\text {term } 1}+\underbrace{\sqrt{\sigma}\left(-\beta(\phi+1)^{2}+\mu+\phi \mu(\phi+2)\right)}_{\text {term } 2}
$$
}

and noting that term 1 is nonnegative for all parameter ranges (see Grafeneder-Weissteiner and Prettner (2009) for details) while term 2 is nonnegative as long as $\mu \geq \beta$.

${ }^{19}$ Figures 1 and 2 are plotted for $\delta=0.05, \rho=0.015, \xi=0.3, \sigma=4$ and $\phi=0.98$. Note the we use different ranges of $\mu$ and $\beta$ to focus on the parameter region where the eigenvalues switch sign. 
Figure 1: Contour plot of eigenvalue 3

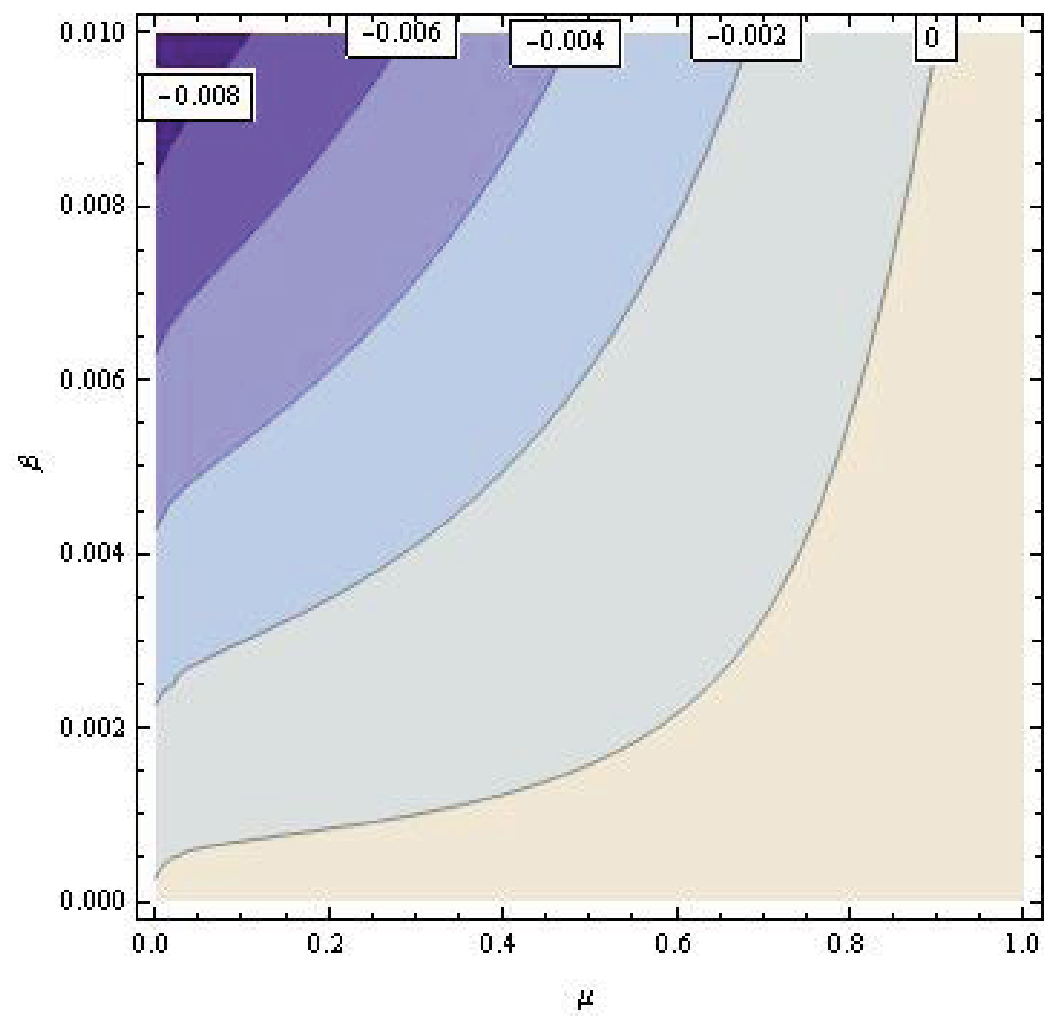


Figure 2: Contour plot of eigenvalue 1

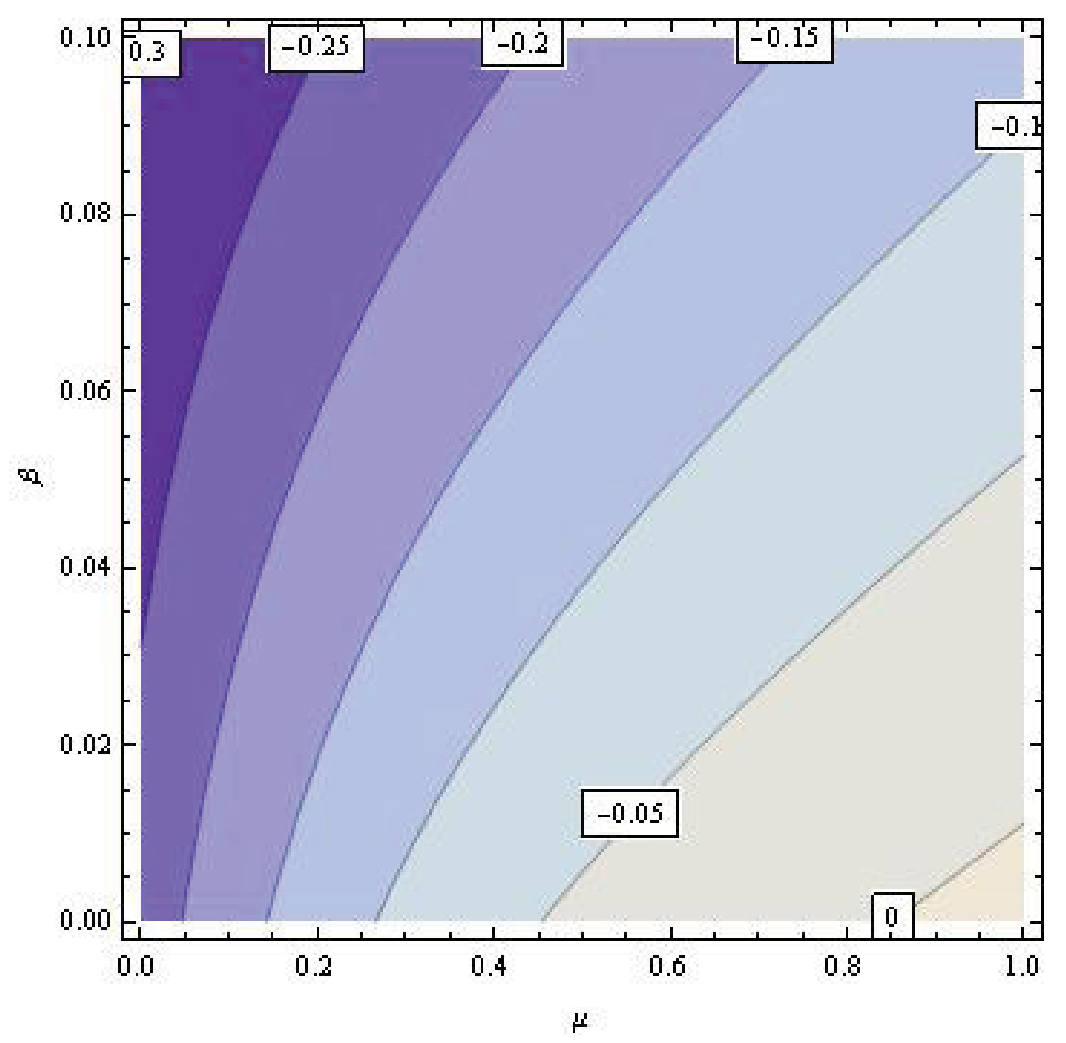


Proposition 1 extends Grafeneder-Weissteiner and Prettner (2009)'s result that with $\beta=\mu$ agglomeration processes crucially depend on the mortality rate. In a setting with nonconstant population size it is both the birth and the mortality rate that determine the stability properties of the symmetric equilibrium. Consequently, in order to fully understand the linkage between demographic change and agglomeration, we must investigate how changes in either of them impact upon agglomeration tendencies. The next section precisely deals with this question.

\subsection{Agglomeration processes in ageing societies}

In the framework with constant population size of Grafeneder-Weissteiner and Prettner (2009), changes in $\mu$ and thus $\beta$ only change the population age structure. With $\beta \neq \mu$, varying either of them, however, also changes the population growth rate. In particular, declines in the birth rate imply both population ageing and a lower population growth rate, whereas changes in the mortality rate leave the mean age unchanged ${ }^{20}$ and only alter population growth (see chapter 7 in Preston et al. (2001)). In order to assess agglomeration tendencies in ageing societies we thus focus on the case of declining birth rates. Moreover, by additionally investigating how the mortality rate impacts upon the stability properties of the symmetric equilibrium, we gain important insights with respect to the population growth based channel on agglomeration.

Figure 1 has already indicated the qualitative effect of declining birth and mortality rates on agglomeration processes. Eigenvalue 3 decreases in the birth rate and increases in the mortality rate. Figures 3 and $4^{21}$ confirm this observation by plotting eigenvalue 3 as a function of trade openness for different $\beta$ and $\mu$. Only for sufficiently low $\beta$ or high $\mu$ we can find levels of economic integration for which eigenvalue 3 gets positive and the symmetric equilibrium becomes unstable. ${ }^{22}$

Declining fertility rates leading to an older population age structure and lower population growth rates at the same time thus destabilize the symmetric equilibrium, i.e. population ageing strengthens agglomeration processes. This is in line with GrafenederWeissteiner and Prettner (2009) who show that with $\beta=\mu$, increasing $\mu$ (and thus also $\beta$ ) above zero (which leads to a younger population structure while leaving the population growth rate unchanged) and thus allowing for a turnover of generations, considerably stabilizes the symmetric equilibrium such that for plausible demographic structures agglomeration processes do not set in. This last observation turns out to also hold in the present

\footnotetext{
${ }^{20}$ This can be easily shown by noting that the proportion of the population at age $t-t_{0}$ is given by $\frac{N\left(t_{0}, t\right)}{N(t)}=\beta e^{-\beta\left(t-t_{0}\right)}$ which is independent of $\mu$. Intuitively, a lower mortality rate on the one hand decreases the tendency to die more quickly and thus makes the population older but on the other hand also increases the population growth rate which exactly offsets the individual ageing effect by making the population younger.

${ }^{21}$ Figures 3 and 4 are plotted for $\delta=0.05, \rho=0.015, \xi=0.3$ and $\sigma=4$. For figure 3 we moreover fix $\mu=0.001$ and for figure 4 we set $\beta=0.001$.

${ }^{22}$ This also holds for the case of population shrinking, since, as figures 1 and 2 indicate, the parameter range within which eigenvalue 1 is positive is a subset of the corresponding one of eigenvalue 3 . Thus eigenvalue 3 is decisive for the stability properties of the symmetric equilibrium even for the case of a shrinking population.
} 
Figure 3: Eigenvalue 3 as a function of $\phi$ for varying $\beta$

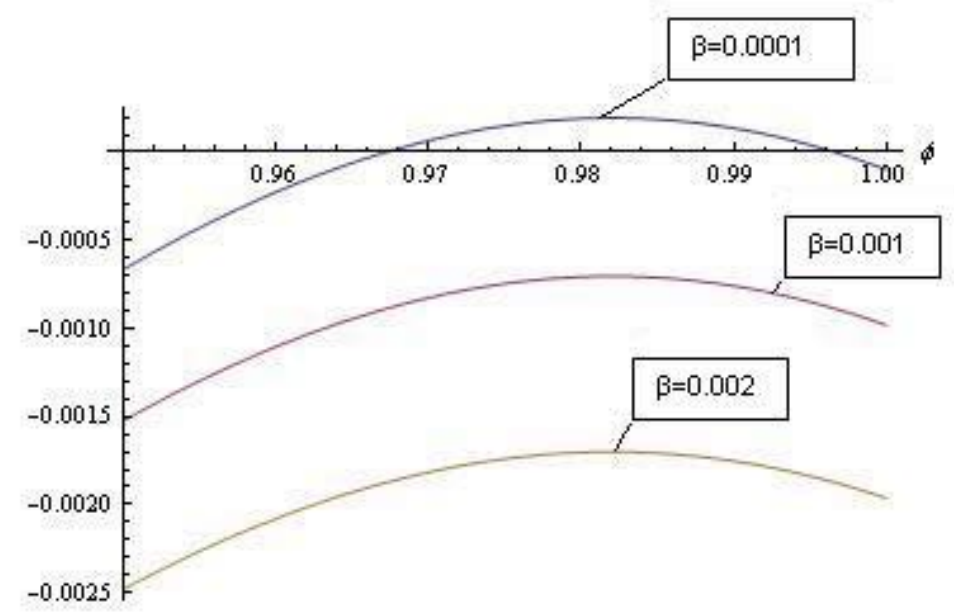

Figure 4: Eigenvalue 3 as a function of $\phi$ for varying $\mu$

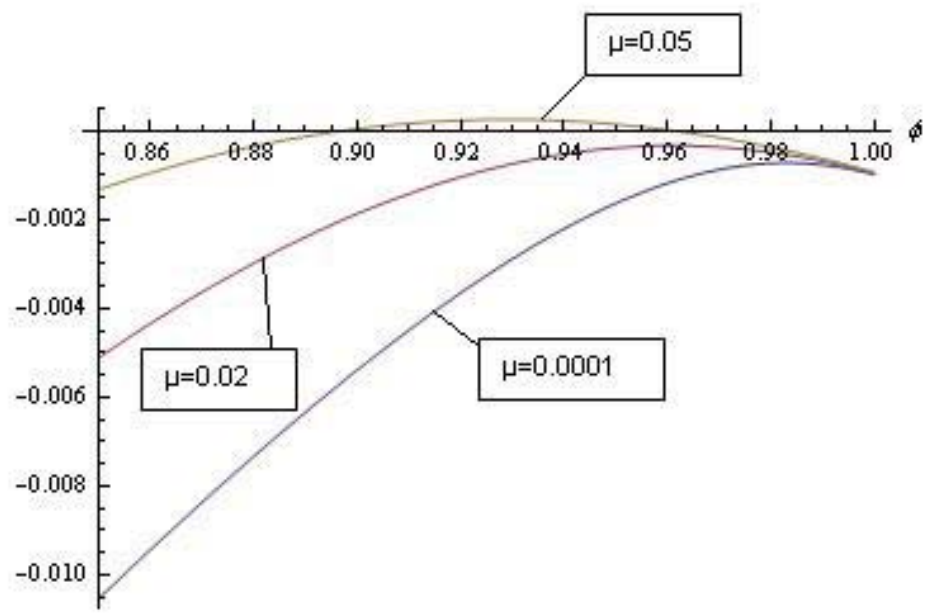


setting with nonconstant population size: for a plausible mortality rate of $\mu=0.0125$ resulting in a life expectancy of 80 years $^{23}$ agglomeration processes never take place as long as $\beta>0.00051 .{ }^{24} \mathrm{~A}$ fertility rate below such a value would clearly be at odds with reality. ${ }^{25}$

While the birth rate mimics the effects of demographic change on agglomeration found in a setting with constant population size, the mortality rate's impact is completely opposite. In particular, lower mortality rates decrease eigenvalue 3 and thus make the symmetric equilibrium more stable. Since changes in the mortality and birth rate affect population growth differently, it is immediate to suspect a population growth based channel to augment the effects of the turnover of generations, which Grafeneder-Weissteiner and Prettner (2009) have identified as the only link between demographic change and agglomeration in a setting with constant population size. Indeed, equation (10) clearly shows that the turnover correction term increases in both demographic parameters implying that declining $\mu$ and $\beta$ should both strengthen agglomeration processes. Lower birth rates, however, decrease the population growth rate, while lower mortality rates increase it. The above findings thus suggest that population growth rate declines as resulting from lower $\beta$ act as an additional agglomeration force, while population growth rate increases due to lower $\mu$ stabilize the symmetric equilibrium. For declining birth rates, the population growth based channel simply reinforces the turnover effect, while it counteracts and even dominates the turnover channel for the case of declining mortality rates. Moreover, by recalling that contemporaneous increases in the birth and mortality rate increase stability (see Grafeneder-Weissteiner and Prettner (2009)), we can also conclude that the birth rate effect dominates the mortality rate effect for the case of constant population size. The next subsection is devoted to verifying this additional population growth based channel on agglomeration processes.

\subsection{The population growth effect}

Since Grafeneder-Weissteiner and Prettner (2009) have shown that with $\beta=\mu$ demographic change only affects the symmetric equilibrium's stability properties via the turnover channel, it is possible to isolate the effects of changes in the population growth rate on agglomeration tendencies by comparing the instability regions of constant to those of varying population size. This is achieved in figure 5 which plots the instability region as a function of the birth rate for the case of zero and the case of positive or negative population growth. ${ }^{26}$ In particular, the borders of the instability region are given by the critical levels of economic integration $\phi_{b r e a k 1}$ and $\phi_{b r e a k 2}$ (see Grafeneder-Weissteiner and Prettner (2009) for details) within which eigenvalue 3 is positive and thus agglomeration processes

\footnotetext{
${ }^{23}$ Since the probability of death during each year equals $\mu$, average life expectancy is $\frac{1}{\mu}$.

${ }^{24}$ We again set $\delta=0.05, \rho=0.015, \xi=0.3, \sigma=4$ for this calculation.

${ }^{25} \mathrm{~A}$ birth rate of $\beta=0.00051$ would imply 0.00051 children per individual.

${ }^{26}$ Note that we now use slightly different parameter values, i.e. $\delta=0.1, \rho=0.1, \xi=0.4, \sigma=2$, to increase the visibility of the population growth effect. For the varying population size case, we fix $\mu$ at 0.003 .
} 
Figure 5: Instability region $\left(\phi_{b r e a k 1}\right.$ and $\left.\phi_{b r e a k 2}\right)$ for constant and varying population size

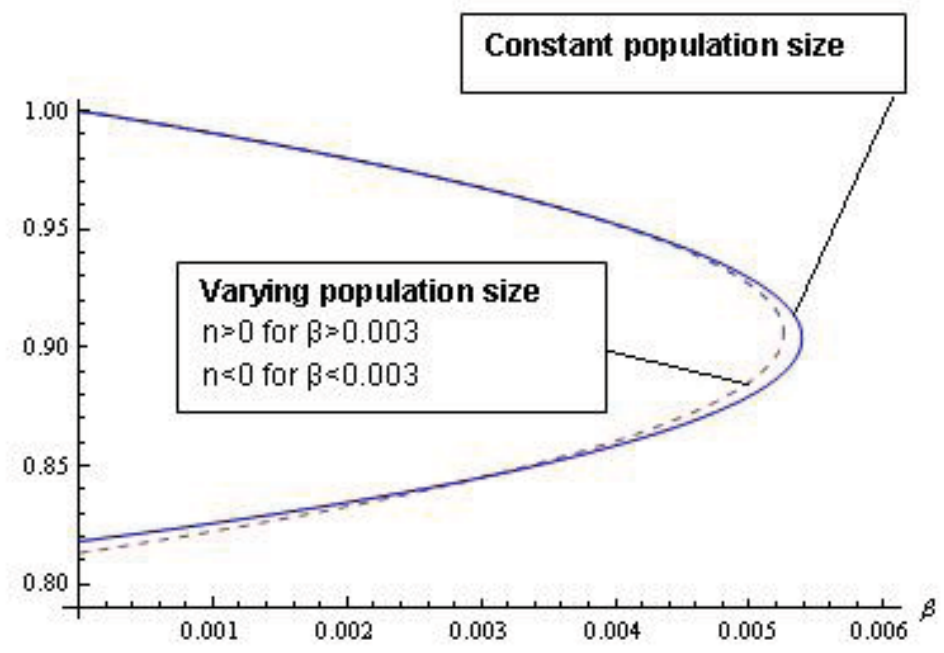

may set in. ${ }^{27}$

Figure 5 confirms our above mentioned presumptions about the effects of changes in the population growth rate. We see that for positive population growth, i.e. $\beta>0.003$, the instability region gets smaller compared to the case of constant population size, whereas it increases for the case of population shrinking, i.e. $\beta<0.003$. This identifies population growth as an additional dispersion force fostering a more equal distribution of productive factors and explaining the differential impact of $\beta$ and $\mu$ on agglomeration processes.

Figure 5 also suggests one qualification to Grafeneder-Weissteiner and Prettner (2009)'s main finding that agglomeration processes are considerably reduced for positive birth and mortality rates. Indeed, for sufficiently strong population shrinking, i.e. $\mu>\beta>0$, the instability region is bigger than for the case of $\mu=\beta=0$ (as represented by the solid line for $\beta=0$ ). Only for the case of a nonnegative population growth rate agglomeration tendencies are thus for sure reduced compared to a setting ignoring demographic structures.

To gain some economic intuition about the particular channel via which population growth impacts upon agglomeration processes, it is useful to reconsider the more informal way of checking the stability properties of the symmetric equilibrium followed by Baldwin

\footnotetext{
${ }^{27}$ Recall that even for the case of population shrinking, eigenvalue 3 is decisive for the stability properties of the symmetric equilibrium since the instability region implicitly defined by eigenvalue 1 is a subset of the instability region defined by eigenvalue 3 .
} 
(1999). This informal way is based on investigating how an exogenous perturbation of the home share of capital, $\theta_{K}$, influences the home capital rental rate relative to the foreign capital rental rate. A positive impact implies instability as capital accumulation would be fostered in the home relative to the foreign region.

Overall, there are four channels via which capital shifting changes the capital rental rate. The first two are the standard anti-agglomerative local competition effect and the pro-agglomerative demand-linked circular causality first introduced by Baldwin (1999). The latter shows that a higher share of capital in one region increases, via higher wealth levels, expenditures and thus operating profits, i.e. capital rental rates, which speeds up capital accumulation. The former, on the other hand, captures the negative impact of agglomeration of capital, i.e. firms, on capital rental rates due to more severe competition (see section 2.3).

In a setting allowing for demographic change in terms of both population ageing and varying population size, two additional forces linked to the economies' demographic parameters $\beta$ and $\mu$ appear. First, there is the anti-agglomerative turnover effect (see again section 2.3). An exogenous rise in the home capital share increases wealth and thus expenditure levels of individuals being currently alive in the home region relative to foreign-based individuals. The negative distributional effects on per capita expenditures resulting from birth and death, i.e. the replacement of dying individuals by newborns whose consumption expenditures are lower since they have zero wealth levels, are thus more pronounced in the home region. This, in turn, decreases the home expenditure share and therefore relative profitability and capital rental rates.

Both higher birth and mortality rates strengthen this first force between demography and agglomeration, which is in sharp contrast to the population growth based channel that additionally affects the linkage between capital shifting and the capital rental for $\beta \neq \mu$. As already indicated in section 2.2, the impact of $\beta$ and $\mu$ in the law of motion of per capita capital is of opposite sign. In particular, equation (11) clearly shows that the market rate of return on capital $\frac{\pi}{w(t) a_{i}}-\delta-\beta+\mu$ depends negatively on $n \equiv \beta-\mu$. The wealth increase due to higher capital income resulting from capital shifting is thus less pronounced the larger is $\mathrm{n}$. This decreased impact upon wealth translates into lower expenditure increases and finally lower increases in the capital rental rate which precisely explains the anti-agglomerative effect of population growth.

\section{Concluding remarks}

The model presented in this paper sheds more light on the linkage between demographic change and agglomeration. In particular, it analyses agglomeration processes in ageing societies and identifies the channels via which demographic developments influence the spatial distribution of economic activity. We extend the framework of Grafeneder-Weissteiner and Prettner (2009), that already incorporates an overlapping generation structure and lifetime uncertainty in the constructed capital model of Baldwin (1999), by additionally al- 
lowing for a nonconstant population size. Changes in either the birth or mortality rate are thus accompanied by changes in the population growth rate. Since population growth per se acts as an important dispersion force and fertility and mortality have opposite effects on this rate, their impacts with respect to the spatial distribution of economic activity also differ. In particular, declining fertility rates strengthen agglomeration processes while declining mortality rates weaken them.

Our framework is suited to assess the possibility of agglomeration tendencies for various demographic developments. Most relevant for industrialized countries is probably the one of declining fertility rates leading to both population ageing and slower population growth. We find that in such a situation agglomeration tendencies are strengthened but still weaker than in a setting that fully ignores demographic structures. In particular, our calibrations suggest that also these countries are currently far away from a situation where catastrophic agglomeration is likely to occur.

Despite the fact that allowing for population growth constitutes one further step toward a more comprehensive understanding of the interrelations between demographic change and agglomeration, many issues still remain open. The above findings e.g. indicate the need for analysing the combined effect of varying birth and mortality rates. In particular, having the opposite effects of birth and mortality rates on agglomeration processes in mind, it is immediate to ask what happens if an economy faces both declining birth and mortality rates. Our results suggest that the answer to this question will crucially depend on the resulting change in the population growth rate and requires a direct comparison of the quantitative effects of changing birth and mortality rates.

Finally, recognizing the tight link between the spatial distribution of economy activity and economic growth perspectives, it is worth investigating how demographic structures impact upon regional growth rates. Since demography has been shown to be of crucial importance for agglomeration, it is immediate to also investigate its effects on the growth impacts of such concentration tendencies. This requires a NEG framework that allows for both demographic change and endogenous long-run growth, a task being on the top of our research agenda.

\section{Acknowledgements}

We thank Harald Fadinger (University of Vienna), Ingrid Kubin (WU), Alexia Prskawetz (Vienna University of Technology), Michael Rauscher (University of Rostock), Gerhard Sorger (University of Vienna), Stefan Wrzaczek (Vienna University of Technology), and the conference participants at the WWTF (Vienna Science and Technology Fund) Workshop

"Agglomeration Processes in Ageing Societies" 2010 for helpful comments and suggestions. The paper was prepared within the research project "Agglomeration Processes in Ageing Societies" funded by the WWTF in its "Mathematics and..." Call 2007. 


\section{Appendix}

\section{A Aggregation over individuals}

\section{A.1 Cohort size}

Since $e^{-\mu\left(t-t_{0}\right)}$ is the probability of an individual of a cohort born at $t_{0}$ to survive to time $t$ and the cohort size of newborns is given by $N\left(t_{0}, t_{0}\right)=\beta N\left(t_{0}\right)$, the size of a cohort born at $t_{0}$ at time $\mathrm{t}$ can be rewritten as

$$
\begin{aligned}
N\left(t_{0}, t\right) & =\beta N\left(t_{0}\right) e^{-\mu\left(t-t_{0}\right)} \\
& =\beta N(0) e^{n t_{0}} e^{-\mu\left(t-t_{0}\right)} \\
& =\beta N(0) e^{(\beta-\mu) t_{0}} e^{-\mu\left(t-t_{0}\right)} \\
& =\beta N(0) e^{\beta t_{0}} e^{-\mu t} \\
& =\beta e^{\beta t_{0}} e^{-\mu t}
\end{aligned}
$$

where we use that population size $\mathrm{N}(\mathrm{t})$ grows with $n=\beta-\mu$ and we normalize $N(0)=1$.

\section{A.2 Aggregate expenditures and capital}

To obtain the laws of motion of per capita expenditures and capital, we must first derive the aggregate consumption rule and the aggregate law of motion of capital. First, note that individual utility maximization yields the individual consumption expenditures rule ${ }^{28}$

$$
e\left(t_{0}, t\right)=(\rho+\mu) a_{i} w(t)\left[k\left(t_{0}, t\right)+h(t)\right]
$$

where

$$
h(t) \equiv \int_{t}^{\infty} \frac{l}{a_{i}} e^{-R^{A}(t, \tau)} d \tau
$$

is human wealth of individuals in capital units, i.e. the present value of lifetime wage income with the annuity factor $e^{-R^{A}(t, \tau)} \equiv e^{-\int_{t}^{\tau}\left(\frac{\pi(s)}{w(s) a_{i}}+\mu-\delta\right) d s}$ used for discounting. Optimal individual consumption expenditures in the planning period t in capital units, $\frac{e\left(t_{0}, t\right)}{a_{i} w(t)}$, are proportional to total wealth with the marginal propensity to consume out of total wealth being constant and equal to the effective rate of time preference $\rho+\mu$. As will become clear soon, it is also necessary to derive an expression for the law of motion of individual human wealth being equal to per capita human wealth. ${ }^{29}$ Applying twice the

\footnotetext{
${ }^{28}$ For details of the derivations see Grafeneder-Weissteiner and Prettner (2009)

${ }^{29}$ With age independency, individual variables of course equal per capita variables, e.g. $\tilde{h}(t)=h(t)$ and $\tilde{l}=l$.
} 
Leibniz rule to equation (31) yields

$$
\begin{aligned}
\dot{\tilde{h}}(t)=\dot{h}(t) & =-\frac{l}{a_{i}}+\int_{t}^{\infty} \frac{l}{a_{i}} e^{-R^{A}(t, \tau)}(-1)\left[-\left(\frac{\pi(t)}{w(t) a_{i}}+\mu-\delta\right)\right] d \tau \\
& =\left(\frac{\pi(t)}{w(t) a_{i}}+\mu-\delta\right) h(t)-\frac{l}{a_{i}} .
\end{aligned}
$$

Using equation (30) in equation (9) yields the aggregate consumption expenditure rule

$$
\begin{aligned}
E(t) & =\beta \int_{-\infty}^{t}(\rho+\mu) a_{i} w(t)\left[k\left(t_{0}, t\right)+h(t)\right] e^{-\mu t+\beta t_{0}} d t_{0} \\
& =\beta(\rho+\mu) a_{i} w(t) e^{-\mu t} \int_{-\infty}^{t}\left[k\left(t_{0}, t\right)+h(t)\right] e^{\beta t_{0}} d t_{0} \\
& =(\rho+\mu) a_{i} w(t)[K(t)+H(t)],
\end{aligned}
$$

where

$$
H(t)=\beta e^{-\mu t} \int_{-\infty}^{t} h(t) e^{\beta t_{0}} d t_{0}=h(t) e^{(\beta-\mu) t}
$$

represents aggregate human wealth.

The law of motion of aggregate capital can be obtained from equation (8) by again applying the Leibniz rule as

$$
\begin{aligned}
\dot{K}(t)= & \beta(\underbrace{k(t, t) e^{(\beta-\mu) t}}_{0}-0)+ \\
& \beta\left[\int_{-\infty}^{t} \dot{k}\left(t_{0}, t\right) e^{\beta t_{0}} e^{-\mu t} d t_{0}+\int_{-\infty}^{t} k\left(t_{0}, t\right) e^{\beta t_{0}} e^{-\mu}(-\mu) d t_{0}\right] \\
= & -\mu K(t)+ \\
& \beta \int_{-\infty}^{t}\left(\frac{w(t) l+\pi(t) k\left(t_{0}, t\right)-e\left(t_{0}, t\right)}{w(t) a_{i}}+(\mu-\delta) k\left(t_{0}, t\right)\right) e^{\beta t_{0}} e^{-\mu t} d t_{0} \\
= & -\mu K(t)+\frac{L(t)}{a_{i}}-\frac{E(t)}{w(t) a_{i}}+\frac{\pi(t)}{w(t) a_{i}} K(t)+\mu K(t)-\delta K(t) \\
= & \left(\frac{\pi(t)}{w(t) a_{i}}-\delta\right) K(t)+\frac{L(t)}{a_{i}}-\frac{E(t)}{w(t) a_{i}},
\end{aligned}
$$

where we used the individual law of motion of capital given in equation (3) to go from the first to the second line. ${ }^{30}$ In contrast to the individual law of motion of capital (3), the aggregate law of motion for capital does not feature the mortality rate, since $\mu K(t)$ just captures the transfer of capital of dying to surviving individuals by the life insurance companies which does not change the rate of return on aggregate capital.

Based on this aggregate law of motion of capital we are now ready to obtain the law

\footnotetext{
${ }^{30}$ Recall also that capital holdings of newborns $\mathrm{k}(\mathrm{t}, \mathrm{t})$ are zero by assumption (no bequests).
} 
of motion of per capita capital as

$$
\begin{aligned}
\dot{\tilde{k}}(t) & =\dot{K}(t) e^{-n t}+K(t) e^{-n t}(-n) \\
& \left.=\left[\left(\frac{\pi(t)}{w(t) a_{i}}-\delta\right) K(t)+\frac{L(t)}{a_{i}}-\frac{E(t)}{w(t) a_{i}}\right)\right] e^{-n t}+K(t) e^{-n t}(-n) \\
& =\frac{\tilde{l}}{a_{i}}-\frac{\tilde{e}(t)}{w(t) a_{i}}+\left(\frac{\pi(t)}{w(t) a_{i}}-\delta\right) \tilde{k}(t)-n \tilde{k}(t) \\
& =\left(\frac{\pi(t)}{w(t) a_{i}}-\delta-\beta+\mu\right) \tilde{k}(t)+\frac{\tilde{l}}{a_{i}}-\frac{\tilde{e}(t)}{w(t) a_{i}} .
\end{aligned}
$$

Finally, the per capita Euler equation is obtained from differentiating the per capita version of the aggregate consumption rule given in equation (33) with respect to time and substituting in the per capita law of motions of capital and human wealth. This yields

$$
\begin{aligned}
\tilde{e}(t)= & (\rho+\mu) a_{i} w(t)[\tilde{k}(t)+\tilde{h}(t)] \\
\dot{\tilde{e}}(t)= & (\rho+\mu) a_{i} w(t)[\dot{\tilde{k}}(t)+\dot{\tilde{h}}(t)] \\
= & (\rho+\mu) a_{i} w(t)\left[\left(\frac{\pi(t)}{w(t) a_{i}}-\delta-n\right) \tilde{k}(t)+\frac{\tilde{l}}{a_{i}}-\frac{\tilde{e}(t)}{w(t) a_{i}}\right]+ \\
& (\rho+\mu) a_{i} w(t)\left[\left(\frac{\pi(t)}{w(t) a_{i}}-\delta+\mu\right) \tilde{h}(t)-\frac{\tilde{l}}{a_{i}}\right] \\
= & (\rho+\mu) a_{i} w(t)\left(-\frac{\tilde{e}(t)}{w(t) a_{i}}\right)+(\rho+\mu) a_{i} w(t)\left(\frac{\pi(t)}{w(t) a_{i}}-\delta\right)[\tilde{k}(t)+\tilde{h}(t)]+ \\
& (\rho+\mu) a_{i} w(t)[-n \tilde{k}(t)+\mu \tilde{h}(t)] .
\end{aligned}
$$

Substituting in $\tilde{e}(t)$ from equation (36), we can rewrite $\dot{\tilde{e}}(t)$ as

$$
\begin{aligned}
\tilde{\tilde{e}}(t)= & (\rho+\mu)(-\tilde{e}(t))+e \tilde{(t)}\left(\frac{\pi(t)}{w(t) a_{i}}-\delta\right)+(\rho+\mu) a_{i} w(t)[-n \tilde{k}(t)+\mu \tilde{h}(t)] \\
= & {\left[\left(\frac{\pi(t)}{w(t) a_{i}}-\delta\right)-(\rho+\mu)\right] \tilde{e}(t)+(\rho+\mu) a_{i} w(t) \mu \tilde{h}(t)-n(\rho+\mu) a_{i} w(t) \tilde{k}(t) } \\
= & {\left[\left(\frac{\pi(t)}{w(t) a_{i}}-\delta\right)-(\rho+\mu)\right] \tilde{e}(t)+(\rho+\mu) a_{i} w(t) \mu\left[\frac{\tilde{e}(t)}{(\rho+\mu) a_{i} w(t)}-\tilde{k}(t)\right] } \\
& -n(\rho+\mu) a_{i} w(t) \tilde{k}(t) \\
= & {\left[\frac{\pi(t)}{w(t) a_{i}}-\delta-\rho\right] \tilde{e}(t)+\left[-\mu(\rho+\mu) a_{i} w(t)(\tilde{k}(t))-n(\rho+\mu) a_{i} w(t) \tilde{k}(t)\right] } \\
= & {\left[\frac{\pi(t)}{w(t) a_{i}}-\delta-\rho\right] \tilde{e}(t)-\beta(\rho+\mu) a_{i} w(t) \tilde{k}(t), }
\end{aligned}
$$

where we used that $\tilde{h}(t)=\frac{\tilde{e}(t)}{(\rho+\mu) a_{i} w(t)}-\tilde{k}(t)$. 
Therefore we have a two dimensional dynamic system of the following form

$$
\begin{aligned}
& \dot{\tilde{e}}(t)=\left[\frac{\pi(t)}{w(t) a_{i}}-\delta-\rho\right] \tilde{e}(t)-\beta(\rho+\mu) a_{i} w(t) \tilde{k}(t) \\
& \dot{\tilde{k}}(t)=\left(\frac{\pi(t)}{w(t) a_{i}}-\delta-\beta+\mu\right) \tilde{k}(t)+\frac{\tilde{l}}{a_{i}}-\frac{\tilde{e}(t)}{w(t) a_{i}}
\end{aligned}
$$

with analogous equations holding in the foreign region.

Note, finally, that by using equation (36) and equation (30) for $t_{0}=t$ the law of motion of per capita consumption expenditures can be rewritten as

$$
\begin{aligned}
\frac{\dot{\tilde{e}}(t)}{\tilde{e}(t)} & =\left[\frac{\pi(t)}{w(t) a_{i}}-\delta-\rho\right]-\beta \frac{\tilde{e}(t)-e(t, t)}{\tilde{e}(t)} \\
& =\frac{\dot{e}\left(t_{0}, \tau\right)}{e\left(t_{0}, \tau\right)}-\beta \frac{\tilde{e}(t)-e(t, t)}{\tilde{e}(t)} .
\end{aligned}
$$

\section{B Derivation of rental rates}

Rental rates given in equations (16) and (17) can be rewritten as ${ }^{31}$

$$
\begin{aligned}
\pi & =\left(\frac{E}{K+\phi K^{*}}+\frac{E^{*} \phi}{\phi K+K^{*}}\right)\left(\frac{\xi}{\sigma}\right), \\
\pi^{*} & =\left(\frac{E^{*}}{K^{*}+\phi K}+\frac{E \phi}{\phi K^{*}+K}\right)\left(\frac{\xi}{\sigma}\right) .
\end{aligned}
$$

By multiplying the nominator as well as the denominator by $e^{-n t}$, we arrive at

$$
\begin{aligned}
\pi & =\left(\frac{\tilde{e}}{\tilde{k}+\phi \tilde{k}^{*}}+\frac{\tilde{e}^{*} \phi}{\phi \tilde{k}+\tilde{k}^{*}}\right)\left(\frac{\xi}{\sigma}\right), \\
\pi^{*} & =\left(\frac{\tilde{e}^{*}}{\tilde{k}^{*}+\phi \tilde{k}}+\frac{\tilde{e} \phi}{\phi \tilde{k}^{*}+\tilde{k}}\right)\left(\frac{\xi}{\sigma}\right) .
\end{aligned}
$$

\section{Intermediate results for the stability analysis}

The Jacobian matrix $J_{\text {sym }}$, which is evaluated at the symmetric equilibrium and given in equation (24), has the following entries $J_{i}$ for $i=1, \ldots, 4$

$$
\begin{aligned}
J_{1}= & \frac{1}{2(\phi+1) \sqrt{\sigma}}\left(\begin{array}{cc}
A(\phi+2)-B \phi & \phi(A+B) \\
\phi(A+B) & A(\phi+2)-B \phi
\end{array}\right), \\
J_{2}= & \left(\begin{array}{cc}
-\frac{a_{i}\left(\phi^{2}+1\right)(A+B)^{2}}{4(\phi+1)^{2} \xi}-\beta a_{i}(\mu+\rho) & -\frac{a_{i} \phi(A+B)^{2}}{2(\phi+1)^{2} \xi} \\
-\frac{a_{i} \phi(A+B)^{2}}{2(\phi+1)^{2} \xi} & -\frac{a_{i}\left(\phi^{2}+1\right)(A+B)^{2}}{4(\phi+1)^{2} \xi}-\beta a_{i}(\mu+\rho)
\end{array}\right), \\
J_{3}= & \frac{1}{a_{i}(\phi+1) \sigma}\left(\begin{array}{cc}
\xi-(\phi+1) \sigma & \phi \xi \\
\phi \xi & \xi-(\phi+1) \sigma
\end{array}\right), \\
J_{4}= & \left(\begin{array}{cc}
\frac{A \phi+\sqrt{\sigma}\left(-\beta(\phi+1)^{2}-\delta\left(\phi^{2}+\phi+1\right)+\mu(\phi+1)^{2}+\phi \rho\right)}{(\phi+1)^{2} \sqrt{\sigma}} & -\frac{\phi(A+B)}{(\phi+1)^{2} \sqrt{\sigma}} \\
-\frac{\phi(A+B)}{(\phi+1)^{2} \sqrt{\sigma}} & \frac{A \phi+\sqrt{\sigma}\left(-\beta(\phi+1)^{2}-\delta\left(\phi^{2}+\phi+1\right)+\mu(\phi+1)^{2}+\phi \rho\right)}{(\phi+1)^{2} \sqrt{\sigma}}
\end{array}\right)
\end{aligned}
$$

\footnotetext{
${ }^{31}$ Note that we suppress time arguments here.
} 
with the parameter clusters $A \equiv \sqrt{\sigma(\delta+\rho)^{2}+4 \beta(\mu+\rho) \xi}$ as well as $B \equiv(\delta+\rho) \sqrt{\sigma}$. 


\section{References}

Baldwin, R. E. (1999). Agglomeration and endogenous capital. European Economic Review, Vol. 43(No. 2):253-280.

Baldwin, R. E., Forslid, R., Martin, P., Ottaviano, G., and Robert-Nicoud, F. (2003). Economic Geography 83 Public Policy. Princeton University Press.

Barro, R. J. and Sala-i-Martin, X. S. (2004). Economic Growth. The MIT Press. Cambridge, Mass.

Buiter, W. H. (1988). Death, birth, productivity growth and debt neutrality. The Economic Journal, Vol. 98:179-293.

Dixit, A. K. and Stiglitz, J. E. (1977). Monopolistic competition and optimum product diversity. American Economic Review, Vol. 67(No. 3):297-308.

Eurostat (2009). Eurostat yearbook 2009. Office for Official Publications of the European Communities.

Fujita, M. and Thisse, J.-F. (2002). Economics of Agglomeration. Cambridge University Press, Cambridge.

Grafeneder-Weissteiner, T. and Prettner, K. (2009). Agglomeration and population aging in a two region model of exogenous growth. Department of Economics Working Papers. Vienna University of Economics and Business Administration.

Preston, S. H., Heuveline, P., and Guillot, M. (2001). Demography. Measuring and Modeling Population Processes. Blackwell Publishing.

The Economist (2009). A special report on ageing populations. The Economist, June 25th 2009.

United Nations (2007). World population prospects: The 2006 revision. Url: http://www.un.org/esa/population/publications/wpp2006/english.pdf.

World Bank (2009). Reshaping Economic Geography. World Development Report 2009.

Yaari, M. E. (1965). Uncertain lifetime, life insurance and the theory of the consumer. The Review of Economic Studies, Vol. 32(No. 2):137-150. 


\section{VIENNA INSTITUTE OF DEMOGRAPHY}

\section{Working Papers}

Buber, Isabella, Parity-specific weights for the Austrian Generations and Gender Survey, VID Working Paper 04/2010.

Testa, Maria Rita, She wants, he wants: Couple's childbearing desires in Austria, VID Working Paper 03/2010.

Buber, Isabella, Wissenschaftlerinnen in Österreich - Zusatzerhebung im Rahmen des GGS.Dokumentation der Datenerhebung und deskriptive Ergebnisse, VID Working Paper 02/2010.

Kuhn, Michael, Stefan Wrzaczek, Alexia Prskawetz, and Gustav Feichtinger, Externalities in a Life-Cycle Model with Endogenous Survival, VID Working Paper 01/2010.

Prettner, Klaus, Population Ageing and Endogenous Economic Growth, VID Working Paper 08/2009.

Št’astná, Anna and Tomáš Sobotka, Changing Parental Leave and Shifts in Second and Third-Birth Rates in Austria, VID Working Paper 07/2009.

Prettner, Klaus and Alexia Prskawetz, Decreasing Fertility, Economic Growth and the Intergenerational Wage Gap, VID Working Paper 06/2009.

Wegner, Christian and Marc Luy, Determinants of General Health and Specific Diseases of Elderly Women and Men: A Longitudinal Analysis for Western and Eastern Germany, VID Working Paper 05/2009.

Wrzaczek, Stefan, Michael Kuhn, Alexia Prskawetz, and Gustav Feichtinger, The Reproductive Value in Distributed Optimal Control Models, VID Working Paper 04/2009.

Wendland, Maike und Isabella Buber, Ein Vergleich der Ergebnisse der ersten Welle des Österreichischen,, Survey of Health, Ageing and Retirement in Europe " (SHARE) und der „Österreichischen Gesundheitsbefragung“ (ATHIS), VID Working Paper 03/2009.

Thomson, Elizabeth, Maria Winkler-Dworak, Martin Spielauer, and Alexia Prskawetz, Union Instability as an Engine of Fertility, VID Working Paper 02/2009.

The Vienna Institute of Demography Working Paper Series receives only limited review. Views or opinions expressed herein are entirely those of the authors. 